\title{
Chronic Neurological Deficits in Mice after Perinatal Hypoxia and Ischemia Correlate with Hemispheric Tissue Loss and White Matter Injury Detected by MRI
}

\author{
Pelin Cengiz $^{a} \quad$ Kutluay Uluc $^{\mathrm{b}, \mathrm{e}}$ Pinar Kendigelen ${ }^{\mathrm{a}, \mathrm{f}}$ Erinc Akture ${ }^{\mathrm{a}, \mathrm{b}}$ \\ Elizabeth Hutchinson $^{c}$ Chihwa Song $^{d}$ Louise Zhang $^{\mathrm{e}}$ Jihae Lee ${ }^{\mathrm{e}}$ \\ Greg E. Budoffe Elizabeth Meyerand ${ }^{d}$ Dandan Sun $^{b, e}$ Peter Ferrazzano ${ }^{a}$ e \\ Departments of a Pediatrics, ${ }^{b}$ Neurological Surgery, ${ }^{c}$ Neurology and d Medical Physics and ${ }^{\text {eW Waisman }}$ \\ Center, University of Wisconsin School of Medicine and Public Health, Madison, Wisc., USA; ${ }^{\mathrm{f}}$ Department of \\ Anesthesiology, Istanbul University Cerrahpaşa Medical School, Istanbul, Turkey
}

\section{Key Words}

Diffusion tensor imaging • Fractional anisotropy •

$\mathrm{Na}^{+} / \mathrm{H}^{+}$exchanger isoform $1 \cdot$ Rotarod $\cdot$ Morris water maze $\cdot$ HOE 642 - Corpus callosum • Myelin basic protein • Neurofilament protein

\begin{abstract}
We investigated the effects of perinatal hypoxia-ischemia (HI) on brain injury and neurological functional outcome at postnatal day (P)30 through P90. HI was induced by exposing P9 mice to $8 \% \mathrm{O}_{2}$ for 55 min using the Vannucci HI model. Following $\mathrm{HI}$, mice were treated with either vehicle control or $\mathrm{Na}^{+} / \mathrm{H}^{+}$exchanger isoform 1 (NHE1) inhibitor HOE 642. The animals were examined by the accelerating rotarod test at P30 and the Morris water maze (MWM) test at P60. $\mathrm{T}_{2}$-weighted MRI was conducted at P90. Diffusion tensor imaging (DTI) was subsequently performed in ex vivo brains, followed by immunohistochemical staining for changes in myelin basic protein (MBP) and neurofilament protein expression in the corpus callosum (CC). Animals at P30 after $\mathrm{HI}$ showed deficits in motor and spatial learning. $T_{2}$ MRI detected a wide spectrum of brain injury in these animals. A positive linear correla-
\end{abstract}

tion was observed between learning deficits and the degree of tissue loss in the ipsilateral hemisphere and hippocampus. Additionally, CC DTI fractional anisotropy (FA) values correlated with MBP expression. Both FA and MBP values correlated with performance on the MWM test. HOE 642-treated mice exhibited improved spatial learning and memory, and less white matter injury in the CC. These findings suggest that $\mathrm{HI}$-induced cerebral atrophy and CC injury contribute to the development of deficits in learning and memory, and that inhibition of NHE1 is neuroprotective in part by reducing white matter injury. $\mathrm{T}_{2}$-weighted $\mathrm{MRI}$ and $\mathrm{DTI}$ are useful indicators of functional outcome after perinatal $\mathrm{HI}$.

Copyright $\odot 2011$ S. Karger AG, Basel

\section{Introduction}

Perinatal hypoxia-ischemia (HI) is a common cause of brain injury resulting in significant mortality and morbidity among both term and preterm neonates [1]. Neurologic outcome in surviving children is variable, ranging from mild cognitive impairment to major debilitating disease [2]. Perinatal HI at different gestational ages re-

\section{KARGER}

(๑) 2011 S. Karger AG, Basel

Fax +4161306 1234

E-Mail karger@karger.ch

www.karger.com
Accessible online at: www.karger.com/dne
Pelin Cengiz, MD

Department of Pediatrics, University of Wisconsin-Madison

School of Medicine and Public Health, Box 4108 Clinical Sciences Center-H6/564 600 Highland Avenue, Madison, WI 53792 (USA)

Tel. +1 608262 2289, E-Mail cengiz@ pediatrics.wisc.edu 
sults in distinct patterns of injury based on region and the cell type $[2,3]$. The mechanisms underlying perinatal HImediated brain injury and their impact on long-term neurological outcomes are not well defined.

The importance of assessing neurobehavioral outcomes in animal studies of cerebral ischemia is now well recognized, and several recent studies have sought to characterize the behavioral and learning deficits in neonatal animals after HI [3-5]. Efforts to correlate injury profiles with behavioral outcome have focused on selective cell death in susceptible brain regions such as the hippocampus and white matter. Early demyelination and subsequent glial scarring are associated with long-term spatial learning and memory deficits in rats after HI [6]. Clinically, MRI and diffusion tensor imaging (DTI) are increasingly used to detect $\mathrm{HI}$-induced brain injury in neonates and to characterize the extent of the injury [2]. A main benefit of the use of MRI in neonates after $\mathrm{HI}$ is its ability to obtain a wide array of clinical information noninvasively, and to follow the evolution of the brain injury longitudinally [7-9].

In the perinatal period, white matter tracts develop from a disorganized state to more organized bundles of white matter tracts [10]. Fractional anisotropy (FA) in DTI reflects a ratio of the water diffusivity along fiber tracts to that across fiber tracts [10]. White matter injury after $\mathrm{HI}$ can result in decreased diffusivity of water along the axonal fibers. This decrease in directional diffusivity can be detected by reductions in FA. While the utility of DTI for assessing white matter injury is well established [9-11], neurobehavioral correlates of DTI findings after $\mathrm{HI}$ are poorly defined.

We have recently reported that pharmacological inhibition of $\mathrm{Na}^{+} / \mathrm{H}^{+}$exchanger isoform 1 (NHE1) activity in postnatal day (P) 9 mice protected hippocampal neurons at 3 days after HI [12]. Neonatal mice that were treated with HOE 642 (a potent and selective inhibitor of NHE1) showed improved motor and spatial learning at P60. However, the long-term effects of NHE1 inhibition after $\mathrm{HI}$ on cerebral atrophy and white matter injury have not been assessed.

In this study, we assessed cerebral atrophy and white matter injury using MRI and immunohistochemistry as well as correlations between MRI findings and behavioral tests of learning and memory. Cerebral atrophy and white matter damage in the corpus callosum (CC) correlated with impaired motor and spatial learning after HI. Inhibition of NHE-1 reduced white matter damage in the CC and improved the animals' performance on behavioral testing.

Chronic Neurological Deficits after Perinatal HI

\section{Materials and Methods}

\section{Materials}

Antibodies against monoclonal anti-myelin basic protein (MBP) and polyclonal anti-neurofilament (NF) were from Millipore (Billerica, Mass., USA) Sigma Inc. HOE 642 was a kind gift from Aventis Pharma (Frankfurt, Germany). Goat anti-mouse Alexa Fluor 488-conjugated IgG and goat anti-rabbit Alexa Fluor 546-conjugated IgG were from Invitrogen (Carlsbad, Calif., USA). The tissue section rinsing solution CitriSolv was from Fisher Inc. (Hampton, N.H., USA) and DPX mounting medium from Sigma Inc.

\section{Induction of Neonatal HI}

C57/B6 mice at P9 were anesthetized with isoflurane (4\% for induction, $1 \%$ for maintenance) plus $30 \% \mathrm{O}_{2}$ and $70 \% \mathrm{~N}_{2}$. The body temperature of the animals was maintained at $37^{\circ} \mathrm{C}$ with a heating pad. Under a surgical microscope, a midline skin incision was made, and the left common carotid artery electrically cauterized as described before [12]. The incision was rinsed with $1 \%$ lidocaine and sutured with a 6-0 Prolene suture. The animals were returned to their dams and monitored continuously for 30 min during a 2-hour recovery period. To induce ipsilateral ischemic injury, the animals were placed in a hypoxia chamber (BioSpherix Ltd., Redfield, N.Y., USA) equilibrated with $8 \% \mathrm{O}_{2}$ and $92 \% \mathrm{~N}_{2}$ at $37^{\circ} \mathrm{C}$ for $55 \mathrm{~min}$. After $\mathrm{HI}$, the animals were monitored continuously for 30 min and then checked every $30 \mathrm{~min}$ for $2 \mathrm{~h}$ and then daily until sacrificed. Sham control animals $[n=5 ; 4$ male $(M), 1$ female $(F)]$ underwent the same surgical procedures except the cauterization of the left common carotid artery and exposure to hypoxia.

\section{Drug Administration}

To selectively inhibit NHE1 with its potent and highly selective inhibitor HOE 642, the animals were randomly divided into HI vehicle control (HI) and HOE 642-treated groups (HI + HOE). HOE $642(0.5 \mathrm{mg} / \mathrm{kg})$ was administered intraperitoneally at 10 $\min , 24$ and $48 \mathrm{~h}$ after $\mathrm{HI}(\mathrm{n}=8 ; 6 \mathrm{M}, 2 \mathrm{~F})$. Due to the short halflife of HOE 642, it was administered at multiple time points (halflife: $40 \mathrm{~min}$ in rats) [13]. The HI vehicle control animals received an equal volume of saline at the same time points $(n=9 ; 6$ F, 3 M).

\section{Accelerating Rotarod Test}

The accelerating rotarod test examines balance and motor coordination as well as skill learning. The accelerating rotarod test was performed when the mice reached P30. After the mice were placed onto a horizontal rotating rod at $4 \mathrm{rpm}$ (Model ENV-575M; Med Associates, St. Albans, Vt., USA), the rotarod was accelerated from 4 to $40 \mathrm{rpm}$ over the course of a 5 -min trial. A single test lasted from the time the mouse was placed on the rotating rod until it fell off or until $5 \mathrm{~min}$ had elapsed. If a mouse grasped the wheel and made 1 complete revolution, the latency to the first complete revolution was recorded [14]. The mouse was then returned to the home cage. Mice were tested for 4 trials per day for 3 consecutive days [15]. The mice rested in their home cages for $20 \mathrm{~min}$ to $1 \mathrm{~h}$ between each trial. The average time on the rotating rod on day 3 is presented.

\section{Hidden Morris Water Maze Test}

Spatial learning and memory were assessed by the Morris water maze (MWM) test in sham, HI and HOE 642-treated mice at 
P60. The mice were trained to locate a hidden escape platform in a water pool as described before [12]. The pool was $124 \mathrm{~cm}$ wide and $45 \mathrm{~cm}$ high. The clear acrylic hidden escape platform was 10 $\mathrm{cm}$ wide and $30 \mathrm{~cm}$ high with a circular top. Distal visual cues were placed on each wall in the room $(441 \times 332 \mathrm{~cm}$, with a background noise of $56 \mathrm{~dB}$ ). The pool was filled with $31 \mathrm{~cm}$ of water $\left(21-23^{\circ} \mathrm{C}\right)$, and illumination at the water level was $21 \mathrm{~lx}$. White and nontoxic paint was added until the water was opaque (about $550 \mathrm{~g}$ of Crayola nontoxic liquid paint). Thus, the platform was hidden by $1 \mathrm{~cm}$ of colored water. Lastly, the pool was virtually divided into equal quadrants with 4 virtual points: west, north, east and south. The escape platform was placed in the center of the northwest quadrant, $26 \mathrm{~cm}$ from the wall at $45^{\circ}$ diagonal from the center.

The mice were transported from the housing room to the testing room. Then, they were allowed to acclimate to the room for 30 min before training or testing. A brief preliminary training was conducted with each mouse to ensure it could swim and climb onto the platform. Each mouse was then trained for a total of 32 trials. That is, each mouse was trained for 8 trials ( 2 blocks) per day for 4 days. Between each trial, the mice were allowed to rest on the platform for $10-15 \mathrm{~s}$. The trials lasted $1 \mathrm{~min}$ or until a mouse found the platform. If the mouse did not find the platform, the mouse was placed on the platform for 10-15 s by the researcher. Latency to reach the platform was automatically tracked and recorded. After trial 32, each animal was given an MWM probe trial, during which the platform was removed and each animal allowed $60 \mathrm{~s}$ to search for the platform in the pool. The amount of time that each animal spent in the trained quadrant and the other quadrants was recorded.

\section{$T_{2}$-Weighted MRI}

MRI was performed using a Varian 4.7-tesla Small Animal MRI scanner with a Varian $200-\mathrm{MHz}$ quadrature mouse radiofrequency coil ( $2.5 \mathrm{~cm}$ internal diameter). For $\mathrm{T}_{2}$ imaging, mice at $\mathrm{P} 90$ after $\mathrm{HI}$ were anesthetized with $1.5 \%$ isoflurane in an oxygen/air mixture administered through a nose cone and then secured in a cradle positioned within the center of the magnet bore. Respiratory rate and body temperature were monitored with an MR-compatible physiology monitoring unit, and temperature was maintained within physiologic limits $\left(37.0 \pm 0.2^{\circ} \mathrm{C}\right)$ using a heated airflow unit. $\mathrm{T}_{2}$-weighted fast spin echo images $(\mathrm{TR}=$ $4,000 \mathrm{~ms}$; effective $\mathrm{TE}=60 \mathrm{~ms}$; echo train length $=8$; matrix size: $128 \times 128$; averages $=40$ ) were acquired from 12 contiguous axial slices with a field of view of $17 \times 17 \mathrm{~mm}$ and a slice thickness of $1 \mathrm{~mm}$. Masks of ipsilateral and contralateral hemispheres, hippocampus and ventricles were created for each slice using ImageJ [16]. Tissue volumes were calculated, and the ventricles and cysts excluded from the hemispheric volume measurement. The volume of hemispheric tissue and hippocampus was expressed as a ratio of ipsilateral to contralateral tissue volumes. 3-D surface reconstructions of the ventricular system were created using the SurfaceGen module in Amira (Visage Imaging Inc., San Diego, Calif., USA).

\section{DTI of ex vivo Brains}

After completion of the $\mathrm{T}_{2}$-weighted MRI scanning, the animals at P90 were anesthetized with isoflurane, transcardially perfused with $4 \%$ paraformaldehyde and decapitated. After postfixation of the brains in $4 \%$ paraformaldehyde overnight, brains were stored in a $30 \%$ sucrose/PBS solution for $48 \mathrm{~h}$ and rinsed with PBS for $24 \mathrm{~h}$. DTI was then performed on ex vivo brain specimens, using a multislice spin echo sequence to acquire 3 reference and 30 noncollinear diffusion-weighted image volumes with the following parameters: $\mathrm{TE} / \mathrm{TR}=22 / 2,000 \mathrm{~ms}$; number of excitations $=4$; matrix size $=192 \times 192$, reconstructed to $256 \times 256$; field of view $=30 \times 30 \mathrm{~mm} ; 25$ slices; slice thickness $=0.5 \mathrm{~mm}$; $\mathrm{b}$-value $=1,100 \mathrm{~s} / \mathrm{mm}^{2}$. FA, mean diffusivity, axial diffusivity and radial diffusivity maps were created using Matlab-based software developed in house.

Regions of interest (ROI) were drawn on each slice, and DTI values calculated for the entire volume of each ROI (fig. 4). For DTI analysis, binary masks of ipsilateral and contralateral CC, hippocampus, basal ganglia and cortex were manually drawn on each of the 25 axial slices, using Amira software. The masks were then applied to the FA and diffusivity maps. Mean values were calculated for the entire volume of each ROI. FA, mean diffusivity, axial diffusivity and radial diffusivity values were analyzed for all ROI and correlated with the behavioral test results.

\section{Double Immunofluorescence Staining}

Ex vivo brains were sectioned ( $35 \mu \mathrm{m}$ thickness) on a freezing sliding microtome (Leica SM2000R; Leica, Ill., USA). The brain sections were either cryoprotected in an antifreeze solution for storage at $-20^{\circ} \mathrm{C}$ or mounted on polylysine-coated slides. In order to quantify white matter injury, 2 coronal brain sections from each brain were used $(0.02 \mathrm{~mm}$ posterior from bregma, interaural $3.82 \mathrm{~mm} ; 0.46 \mathrm{~mm}$ posterior from bregma, interaural $3.82 \mathrm{~mm}$ ) [17]. After rinsing with Tris-buffered saline (TBS), brain sections were incubated with TBS++ $(0.1 \%$ Triton X-100 and $3 \%$ goat serum in $0.1 \mathrm{M} \mathrm{TBS}$ ) for $30 \mathrm{~min}$ at $37^{\circ} \mathrm{C}$. Sections were doublestained with monoclonal antibody against MBP (1:50) and rabbit polyclonal antibody against NF (1:160) for $1 \mathrm{~h}$ at $37^{\circ} \mathrm{C}$ and then overnight at $4^{\circ} \mathrm{C}$. After washing with TBS $(3 \times 10 \mathrm{~min})$, brain sections were incubated for $1 \mathrm{~h}$ at $37^{\circ} \mathrm{C}$ with goat anti-mouse $\mathrm{Al}-$ exa Fluor 488-conjugated IgG for MBP (1:200) and goat anti-rabbit Alexa Fluor 546-conjugated IgG for NF (1:200). Following rinsing with TBS, sections were incubated in TO-PRO-3 Iodide (1:1,500 in TBS++) for 15 min and mounted on slides. The slides were imaged with a Leica DM IRE 2 inverted confocal microscope and Leica microscope software (Leica Inc., Ill., USA). For quantification of MBP and NF immunofluorescence staining, images were autothresholded using ImageJ to subtract background staining. ROI were selected within the ipsilateral and contralateral CC. Fluorescence intensity was calculated as the percentage of fluorescence-positive pixels within each ROI, and expressed as a ratio of the ipsilateral to contralateral values. CC thickness was measured on coronal sections in each brain at 3 different locations (fig. 3c). The mean thickness of the ipsilateral CC was expressed as a ratio of ipsilateral to contralateral values.

\section{Statistical Analysis}

Values are expressed as means \pm SD. The Mann-Whitney rank sum test was used to compare the vehicle control and drugtreated groups. ANOVA with Tukey's post test was used in the case of multiple comparisons. $\mathrm{p} \leq 0.05$ were considered statistically significant. Pearson correlation analysis and linear regression were performed, with the significance of the correlation determined by ANOVA. Statistical analysis was performed using PASW Statistics 18 (SPSS Inc., Chicago, Ill., USA). 
Fig. 1. $\mathrm{T}_{2}$-weighted MRI revealing variable degrees of HI-induced hemispheric damage. $\mathrm{IL}=$ Ipsilateral; $\mathrm{CL}=$ contralateral. a Representative $\mathrm{T}_{2}$-weighted images showing the spectrum of brain lesions after HI (mild, moderate and severe; $\mathrm{n}=14$ ). $\mathrm{T}_{2}$-weighted MRI was performed at $\mathrm{P} 90$ after induction of HI in mice at P9. b Representative 3-D reconstructions of the cerebral ventricles after HI. Arrows: normal ventricles. Arrowheads: ventriculo-megaly. c Hemispheric tissue volume measured in sham, HI and HOE 642-treated animals. Representative ROI for hemispheric tissue volume calculation are shown for IL (horizontal line fill) and CL hemispheres (vertical line fill). Ventricles and cysts were excluded, and the volume of hemispheric tissue was expressed as a ratio of ipsilateral to contralateral tissue volume. d Hippocampal tissue volume ratios in sham, HI and $\mathrm{HI}+\mathrm{HOE}$ animals are shown. Representative ROI for hippocampal volume calculation are depicted. $\mathbf{c}, \mathbf{d}^{*} \mathrm{p}<0.05$.

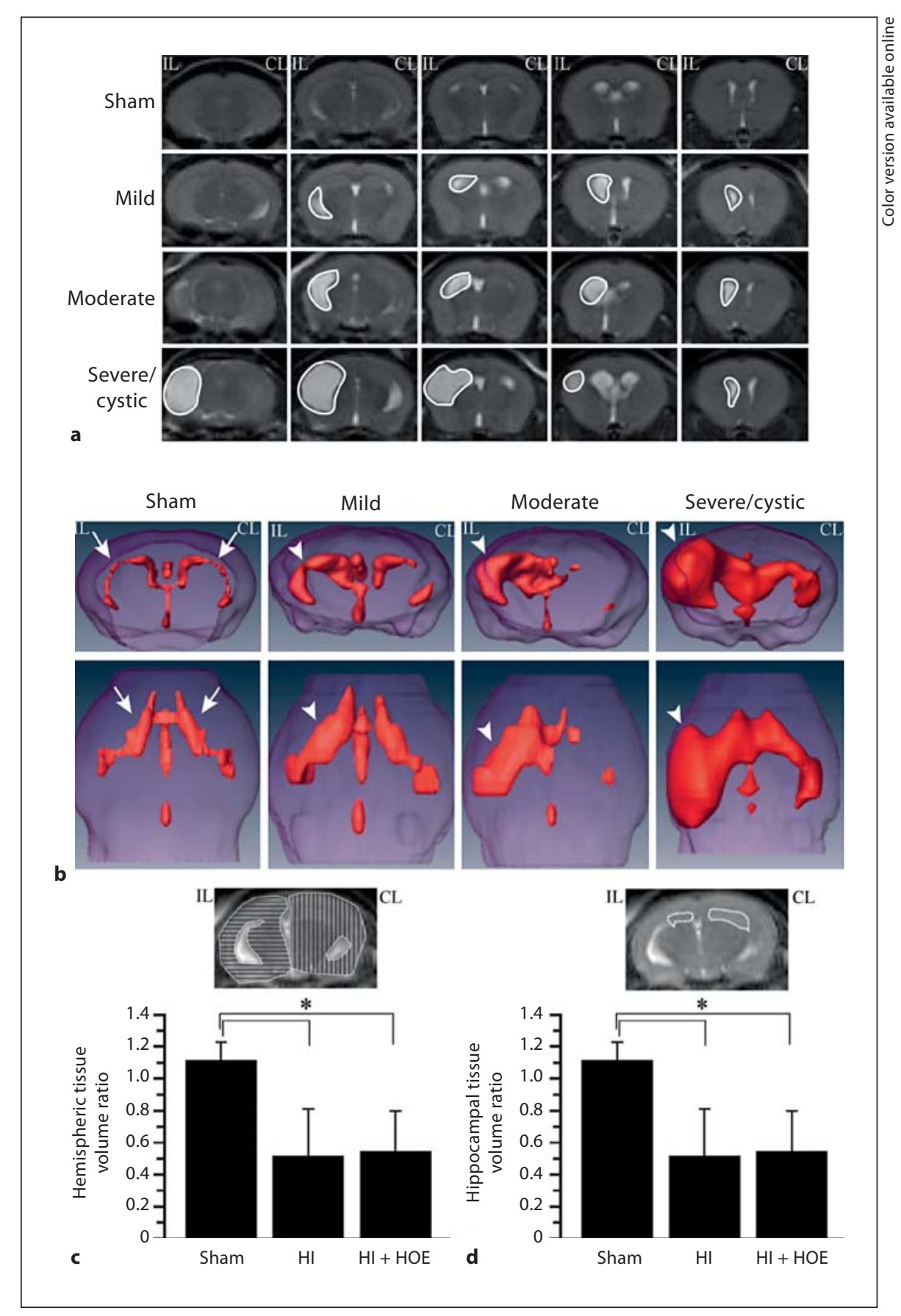

\section{Results}

\section{$T_{2}$-Weighted MRI Reveals Variable Degrees of Brain}

Injury in HI + HOE-Treated Mice after Perinatal HI

$\mathrm{T}_{2}$-weighted MRI demonstrated a wide spectrum of brain injury at P90 (fig. 1a), with mild injury ( $<15 \%$ tissue volume loss), moderate injury (15-30\% tissue volume loss) and severe injury/cysts. Moreover, 3-D reconstructions of the ventricular system illustrate that cerebral tissue loss was accompanied by a similar degree of ventriculomegaly, with formation of large cysts in the most severely injured brains (fig. 1b). Mild injury was seen in 4 $\mathrm{HI}$ and $4 \mathrm{HI}+\mathrm{HOE}$ animals, moderate injury was seen in $4 \mathrm{HI}$ and $2 \mathrm{HI}+\mathrm{HOE}$ animals, and severe injury/cysts 


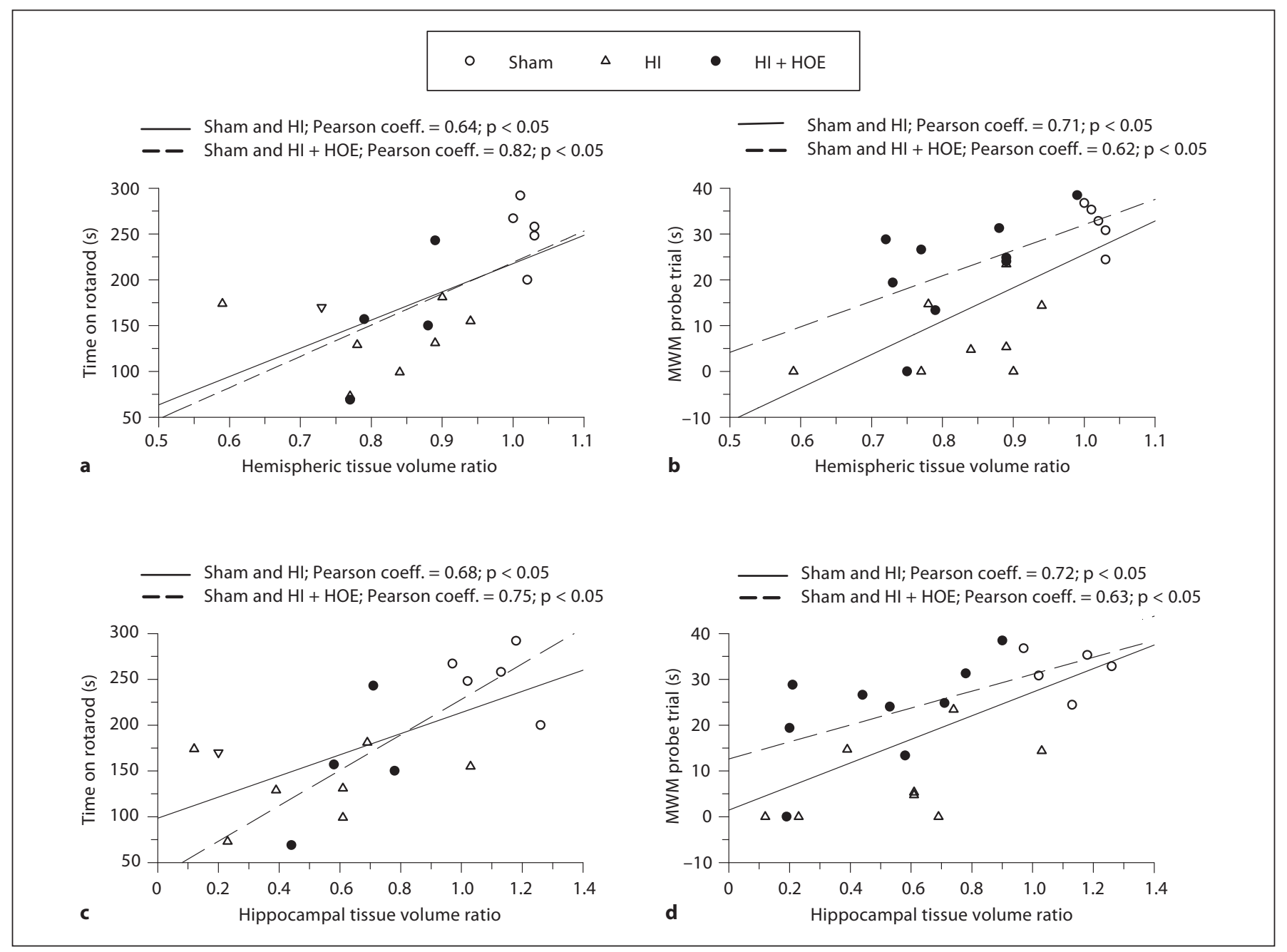

Fig. 2. Correlation of ipsilateral hemispheric tissue volume and hippocampal tissue volume with motor and spatial learning in mice after HI. The accelerating rotarod test and MWM probe trial were performed at P30 and P60 after HI, respectively. Hemispheric and hippocampal tissue volume ratios of these animals were determined by $\mathrm{T}_{2}$-weighted $\mathrm{MRI}$ at $\mathrm{P} 90$. Correlations are presented for sham/HI and sham/HI + HOE animals. a Correla-

were detected in $1 \mathrm{HI}$ and $2 \mathrm{HI}+\mathrm{HOE}$ animals. Figure $1 \mathrm{c}$ shows that the ipsilateral-to-contralateral hemispheric tissue volume ratios were significantly reduced in the HI group, compared with the sham control. A loss of ipsilateral hippocampal tissue volume was also detected in $\mathrm{HI}$ brains (fig. 1d). The hemispheric and hippocampal tissue volume ratios were similar in the $\mathrm{HI}$ and $\mathrm{HI}+\mathrm{HOE}$ groups (fig. 1c, d). These data are consistent with previous reports that developing immature brains exhibit variable responses to $\mathrm{HI}$, both in rodent models and clinically [18, 19].

tion of ipsilateral hemispheric tissue volume ratio with time spent on the rotarod. b Correlation of ipsilateral hemispheric tissue volume ratio with time spent in the training quadrant (MWM probe trial). c Correlation of ipsilateral hippocampal tissue volume ratio with time spent on the rotarod. d Correlation of ipsilateral hippocampal tissue volume ratio with time spent in the training quadrant (MWM probe trial).

\section{Ipsilateral Hemispheric and Hippocampal Tissue Volume Loss Correlates with Motor and Spatial Learning after Perinatal HI}

We have previously shown that induction of HI in these animals at $\mathrm{P} 9$ resulted in impaired motor learning as measured by the accelerating rotarod test, and impaired spatial learning and memory as measured by the MWM test [12]. In the current study, Pearson correlation and linear regression analyses demonstrated that the degree of hemispheric and hippocampal tissue volume loss in both HI and HOE 642 animals correlated with their 


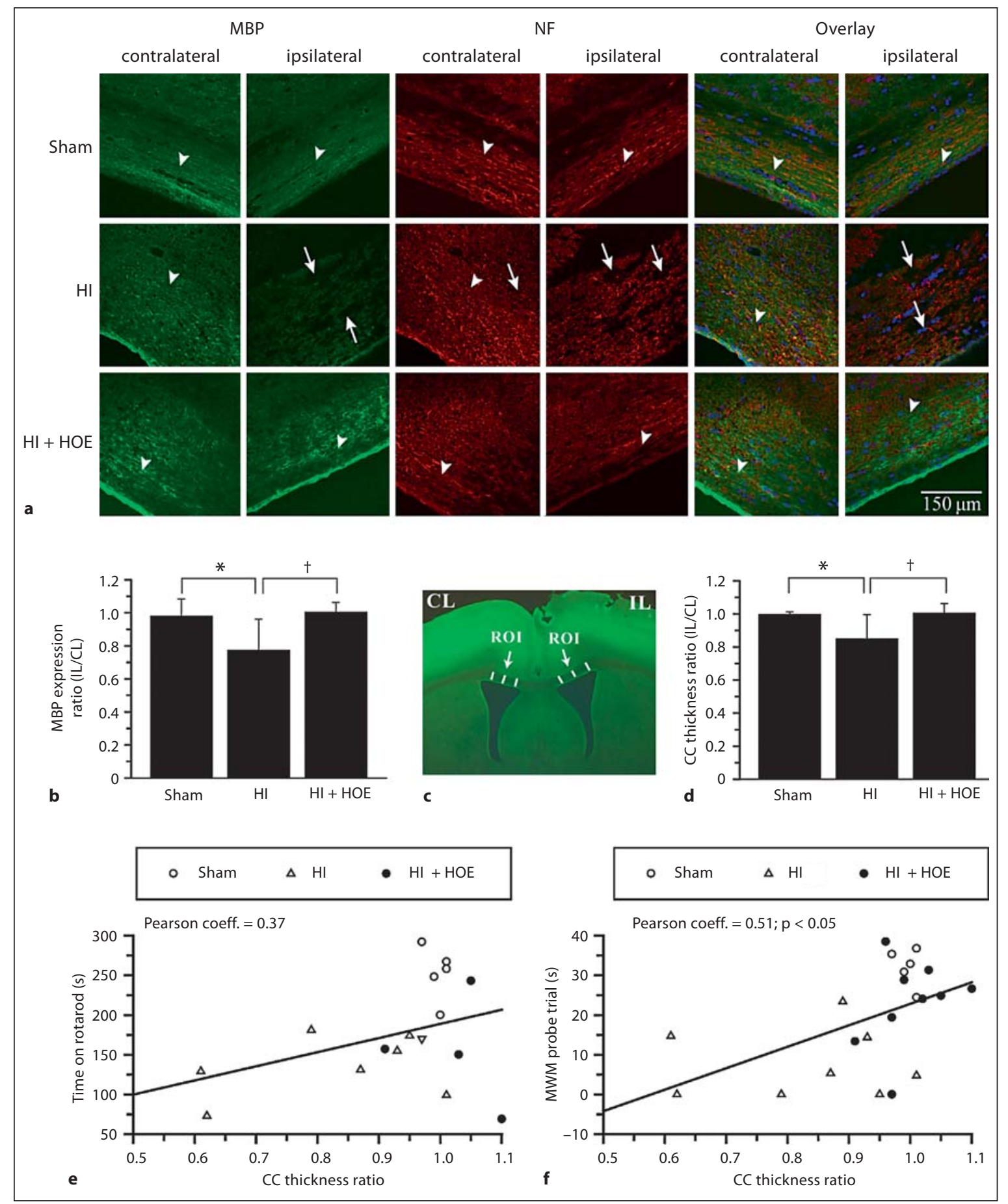

Fig. 3. HI-induced loss of MBP and NF expression in the ipsilateral CC. Changes in MBP and NF protein expression after HI were examined by immunohistochemical staining in the contralateral and ipsilateral CC of ex vivo brains at P90 after HI. CL = Contralateral; $\mathrm{IL}=$ ipsilateral. ${ }^{*} \mathrm{p}<0.05$ sham vs. $\mathrm{HI} ;{ }^{\dagger} \mathrm{p}<0.05$ $\mathrm{HI}$ vs. HI + HOE. a Representative images of MBP and NF stainings are shown from each group. Arrowheads: abundant MBP and NF expression. Arrows: reduced MBP and NF expression. TO-PRO-3 Iodide used for nuclear staining. $\mathbf{b}$ MBP expression is expressed as the ratio of ipsilateral to contralateral values in sham, HI and HI + HOE animals $(\mathrm{n}=5-9)$. c Three ROI in the CC were selected and the thickness of the CC measured in each brain and expressed as a mean value. d CC thickness was expressed as the ratio of the ipsilateral to contralateral mean values in sham, $\mathrm{HI}$ and $\mathrm{HI}+\mathrm{HOE}$ animals. e Correlation of CC thickness ratio with time spent on rotarod. $\mathbf{f}$ Correlation of CC thickness ratio with time spent in the training quadrant (MWM probe trial). 
performance on the accelerating rotarod test (fig. 2a, c). Similarly, changes in hemispheric and hippocampal tissue volume ratios linearly correlated with the performance on the MWM probe trial in HI and HOE 642-treated animals (fig. 2b, d). However, the shallower regression slopes in HOE 642-treated animals imply that these animals spent more time in the training quadrant despite their similar degree of hemispheric and hippocampal tissue volume loss (fig. 2b, d). This suggests that NHE1 inhibition improved spatial learning and memory independent of hemispheric and hippocampal tissue volume loss.

\section{NHE1 Inhibition Preserves CC MBP Expression,}

CC Thickness and Neurobehavioral Outcome after Perinatal HI

To investigate white matter injury in these animals, and its potential contribution to the neurobehavioral outcome, we evaluated the expression of MBP and NF in the ipsilateral and contralateral CC by immunohistochemical staining in sham, HI and HOE 642-treated animals at P90. As shown in figure $3 \mathrm{a}$, the contralateral and ipsilateral CC in sham brains exhibited normal fiber tracts with abundant MBP and NF immunofluorescence signals (arrowheads). In contrast, the ipsilateral CC of HI brains expressed less MBP as well as less NF immunostaining (arrows). The overlay images of MBP and NF immunostaining revealed loss of MBP and NF expression in the ipsilateral CC after HI (fig. 3a, arrows). Most importantly, HOE 642-treated brains exhibited significantly more MBP immunostaining in the ipsilateral CC than the HI controls $(\mathrm{p}<0.05$; fig. 3a, b). The fiber tract morphology of the ipsilateral CC in the HOE 642-treated brains was similar to that of the ipsilateral CC of sham brains (fig. 3a, arrowheads). Although NF expression was decreased in HI animals, this did not reach statistical significance (data not shown). Measurement of CC thickness revealed that there was a significant reduction in the $\mathrm{HI}$ brains at P90 (fig. 3c, d). In contrast, the CC thickness of the HOE 642 -treated brains was similar to that of the sham controls (fig. 3d). Furthermore, there was a positive linear relation between CC thickness and motor and spatial learning when all groups were pooled for correlation analysis (fig. 3e, f). The nonpooled individual groups did not illustrate correlations (data not shown). This could be explained by the clustered data distribution or small sample size. Taken together, these data suggest that induction of $\mathrm{HI}$ in mice at P9 led to a sustained loss of MBP and axonal tract degeneration in the $\mathrm{CC}$ at $\mathrm{P} 90$. Inhibition of NHE1 after HI attenuated the injury in the CC and improved neurobehavioral outcome.
Correlation of Ipsilateral CC FA Values with Loss of

$M B P$ Expression and Spatial Learning Deficits

We further investigated the white matter damage in the ipsilateral CC by DTI analysis. Representative FA maps and ROI are shown in figure 4a. FA values for the sham, HI and HI + HOE groups were $0.48 \pm 0.06,0.44$ \pm 0.08 and $0.46 \pm 0.07$, respectively $(\mathrm{p}>0.05)$. However, a linear correlation between the ipsilateral CC FA value and MBP immunostaining values (in the pooled groups) was significant $(R=0.43 ; p<0.05)$, suggesting that the reduction in FA values in the ipsilateral CC after HI represents a loss of myelinated axons after HI (fig. 4b). No changes were found in axial or radial diffusivity values among these groups. We speculated that the damage in the ipsilateral CC may play a role in neurobehavioral deficit formation. A positive correlation between the ipsilateral CC FA values and the time spent in the training quadrant of the MWM probe trial was detected $(R=0.45$; $\mathrm{p}<0.05$; fig. $4 \mathrm{c}$ ). Collectively, these findings suggest that the decreases in CC FA values reflect a degeneration of the myelinated axonal tracts in the CC after $\mathrm{HI}$, and that white matter injury may contribute to spatial learning and memory deficits in mice after HI.

\section{Discussion}

\section{$T_{2}$-Weighted MRI Reveals Variable Degrees of Brain} Injury after Perinatal HI

In the current study, a heterogeneous injury was detected in mice at $\mathrm{P} 90$ after perinatal $\mathrm{HI}$ using $\mathrm{T}_{2}$-weighted MRI. Moderate-to-severe brain injury was evident with varying degrees of hemispheric tissue volume loss and ventriculomegaly. The most severe injuries presented as cyst formation with cavitation in the posterior periventricular white matter. This wide spectrum of brain damage is characteristic of immature brains in response to $\mathrm{HI}$, and is consistent with both preclinical studies with rodent $\mathrm{HI}$ models and clinical findings in children after neonatal HI $[1,2,5,18,19]$. The heterogeneity of HI-induced brain injuries results from selective vulnerability of specific populations of neurons and oligodendrocytes to ischemic insult in the developing brain $[2,19]$. Moreover, age-dependent differences in brain repair processes such as neurogenesis, oligodendrocyte regeneration and inflammation may also contribute to specific anatomic patterns of injury [12]. Gender-dependent differences also play an important role after HI [20]. In the current study, the sample size for each gender was small and did not allow us to investigate effects of gender on cerebral 

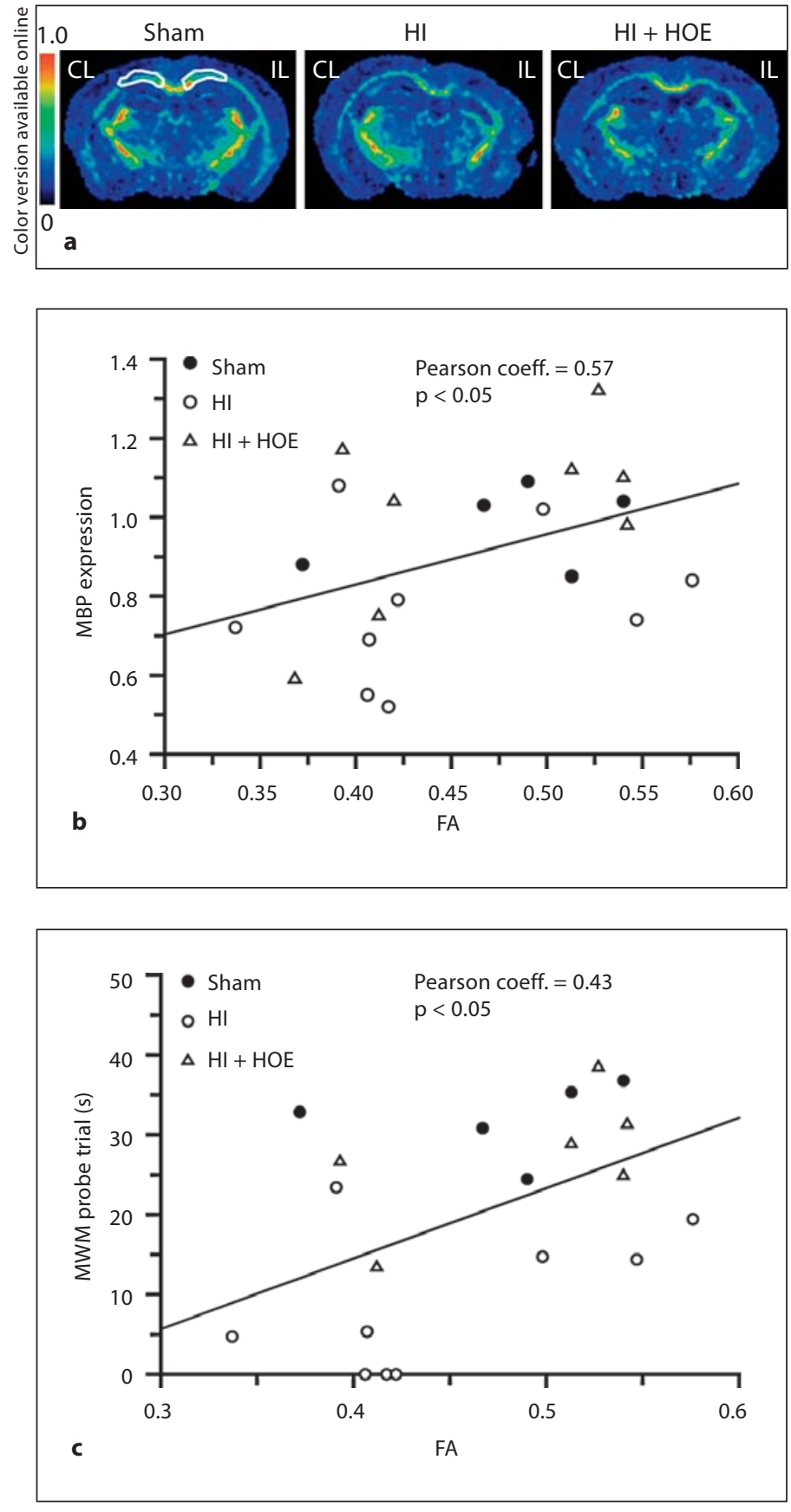

Fig. 4. Correlation of ipsilateral CC FA values with MBP protein expression and MWM probe trial. DTI was performed on ex vivo brains at $\mathrm{P} 90$ in sham, $\mathrm{HI}$ and $\mathrm{HI}+\mathrm{HOE}$ groups after perinatal $\mathrm{HI}$ induced at P9. $\mathrm{CL}=$ Contralateral; $\mathrm{IL}=$ ipsilateral. a Representative FA maps from each group demonstrating ROI selection. b Correlation of CC FA values with MBP protein expression in SHAM, HI and HI + HOE animals. MBP immunofluorescence intensity was presented as the ratio of ipsilateral to contralateral CC intensity. $\mathrm{R}=0.43 ; \mathrm{p}<0.05$. c Correlation of CC FA values with MWM probe trial in sham, $\mathrm{HI}$ and $\mathrm{HI}+\mathrm{HOE}$ animals. $\mathrm{R}=$ $0.45 ; \mathrm{p}<0.05 . \mathrm{N}=11$. $\mathbf{b}, \mathbf{c}$ Regression line represents the mean of all animals studied.

Chronic Neurological Deficits after Perinatal HI atrophy and behavioral deficits in each group. Thus, future study is needed to address this issue.

\section{HI-Induced White Matter Damage in Ipsilateral CC}

Using the immunofluorescence staining approach, we observed a significant reduction in MBP expression (fig. 3b) in the ipsilateral CC after HI. We also detected a reduction of NF expression and axonal disorganization after HI. FA is a measure of the directionality of water diffusion within tissue, and is a sensitive indicator of white matter injury $[18,21]$. HI caused FA reduction (approx. 7\%), which may reflect $\mathrm{HI}$-induced neuronal damage, demyelination and loss of axon fibers in the ipsilateralCC. This is consistent with findings by others regarding HI-mediated white matter damage. At P70 after HI, rats exhibited a significantly lower FA in the ipsilateral $\mathrm{CC}$, proximal external capsule and anterior commissure [22]. Moreover, a similar decrease in FA in the CC has been detected in rats at P40-50 after chronic sublethal hypoxia [23]. We also observed a significant reduction in CC thickness in HI animals at P90. A similar finding was reported in rats at $\mathrm{P} 90$ in a global perinatal asphyxia model [24]. It has been documented that P0-50 in rats is an important period for myelination, maturation and pruning of axonal tracts in the CC, suggesting that animals are susceptible to white matter injury from HI during this vulnerable period [24]. Our findings demonstrate that $\mathrm{HI}$ induction in $\mathrm{P} 9$ mice could cause a sustained injury to white matter at $\mathrm{P} 90$.

\section{Contribution of Hemispheric Tissue Volume Loss and} White Matter Damage to Motor Learning and Spatial Learning Deficits

In the current study, we found that decrease in ipsilateral hemispheric tissue volume positively correlated with motor and spatial learning deficits in mice after HI. However, inhibition of NHE1 protein with HOE 642 did not reduce the hemispheric tissue volume loss at P90, but significantly improved motor learning and memory at P60 (the latter has previously been reported [12]). It has been reported that neuronal plasticity after HI can improve memory loss without reducing tissue atrophy in either hippocampus or cortex [25]. Treatment with HOE 642 was shown to be neuroprotective of the hippocampus 3 days after $\mathrm{HI}$ in neonatal mice [12]. In addition to the possible effects of HOE 642 on neuronal plasticity, we speculate that less white matter damage may also have contributed to better functional outcome in the HOE 642-treated mice. Myelin plays an important role in learning and memory function $[6,24,26]$. In our study, white matter 
damage in the CC (DTI FA values, MBP staining and CC thickness measurement) correlated with the performance of animals on motor learning and memory tests. Moreover, HOE 642-treated animals exhibited a reduced loss of MBP and thicker ipsilateral CC compared with HI animals. Damage or agenesis of the CC in children is associated with attention deficit disorder and developmental coordination disorder $[27,28]$. Both of these clinical disorders are characterized by impairments of posture, motor coordination and sensorimotor coordination [27]. Therefore, the association of impaired motor learning with CC injury in our study may reflect a phenomenon similar to the motor coordination impairment in humans. However, we could not completely rule out the contribution of the hippocampus to memory deficits in this study because we only assessed hippocampal damage by $\mathrm{T}_{2}$-weighted MRI. Our findings suggest that hemispheric tissue volume and white matter injury are involved with impairments of memory and learning, and that the improved memory and learning seen in the HOE 642 -treated animals may in part result from a reduction in white matter injury.

Inflammation and gliosis play an important role in white matter injury after perinatal HI [29-32]. Although preterm neonates are more susceptible to white matter injury after $\mathrm{HI}$ than term neonates, term neonates do show white matter injury which is associated with motor, perceptual, behavioral and cognitive disorders [33]. NHE1 is highly expressed in reactive astrocytes 3 days after $\mathrm{HI}$ in $\mathrm{P} 9$ mice [12]. In addition, we found that NHE1 inhibition with HOE 642 decreases microglial activation after lipopolysaccharide- or hypoxia-mediated stimulation [34]. It has been shown that minocycline-mediated suppression of neuroinflammation improves cognitive, learning and memory deficits after HI [35]. Taken together, our data imply that HOE 642 could reduce white matter injury and improve behavioral outcomes by decreasing neuroinflammation mediated by astrocytes and microglia.

In summary, in the present study, we investigated the effects of $\mathrm{HI}$ in $\mathrm{P} 9$ mice on the development of white matter injury and long-term functional outcome at P30 through P90. A positive linear correlation was observed between cerebral atrophy, white matter damage and neurological functional deficits. In particular, our data suggest that $\mathrm{HI}$-induced ipsilateral cerebral atrophy and CC injury are associated with motor and spatial learning deficits. NHE1 inhibition with HOE 642 decreases the CC injury and improves spatial learning and memory. Combined $\mathrm{T}_{2}$-weighted MRI and DTI are useful for evaluating HI-mediated injury to gray and white matter after HI, and for correlation with long-term functional outcome.

\section{Acknowledgments}

This study was supported in part by a University of Wisconsin Department of Pediatrics Research and Development Grant (P.C.), grant 1UL1RR025011 from the Clinical and Translational Science Award program of the National Center for Research Resources and NIH (P.F.), and NIH grants R01NS38118 and R01NS48216 (D.S.) and NIH P30 HD03352 (Waisman Center).

\section{References}

$\checkmark 1$ Ferriero DM: Neonatal brain injury. N Engl J Med 2004;351:1985-1995.

$\checkmark 2$ Ferriero DM, Miller SP: Imaging selective vulnerability in the developing nervous system. J Anat 2010;217:429-435.

3 Fan LW, Lin S, Pang Y, Lei M, Zhang F, Rhodes PG, Cai Z: Hypoxia-ischemia induced neurological dysfunction and brain injury in the neonatal rat. Behav Brain Res 2005; $165: 80-90$.

-4 Arteni NS, Pereira LO, Rodrigues AL, Lavinsky D, Achaval ME, Netto CA: Lateralized and sex-dependent behavioral and morphological effects of unilateral neonatal cerebral hypoxia-ischemia in the rat. Behav Brain Res 2010;210:92-98.

5 Vannucci RC, Vannucci SJ: Perinatal hypoxic-ischemic brain damage: evolution of an animal model. Dev Neurosci 2005;27:81-86.
6 Huang Z, Liu J, Cheung PY, Chen C: Longterm cognitive impairment and myelination deficiency in a rat model of perinatal hypoxic-ischemic brain injury. Brain Res 2009; 1301:100-109.

7 Widerøe M, Olsen Ø, Pedersen TB, Goa PE, Kavelaars A, Heijnen C, Skranes J, Brubakk AM, Brekken C: Manganese-enhanced magnetic resonance imaging of hypoxic-ischemic brain injury in the neonatal rat. Neuroimage 2009;45:880-890.

$\checkmark 8$ van de Looij Y, Chatagner A, Hüppi PS, Gruetter R, Sizonenko SV: Longitudinal MR assessment of hypoxic ischemic injury in the immature rat brain. Magn Reson Med 2011; 65:305-312.
9 Miller SP, Vigneron DB, Henry RG, Bohland MA, Ceppi-Cozzio C, Hoffman C, Newton N, Partridge JC, Ferriero DM, Barkovich AJ: Serial quantitative diffusion tensor MRI of the premature brain: development in newborns with and without injury. J Magn Reson Imaging 2002; 16:621-632.

10 Drobyshevsky A, Derrick M, Wyrwicz AM, Ji X, Englof I, Ullman LM, Zelaya ME, Northington FJ, Tan S: White matter injury correlates with hypertonia in an animal model of cerebral palsy. J Cereb Blood Flow Metab 2007;27:270-281.

11 Stone BS, Zhang J, Mack DW, Mori S, Martin LJ, Northington FJ: Delayed neural network degeneration after neonatal hypoxia-ischemia. Ann Neurol 2008;64:535-546. 
12 Cengiz P, Kleman N, Uluc K, Kendigelen P, Hagemann T, Akture E, Messing A, Ferrazzano P, Sun D: Inhibition of $\mathrm{Na}^{+} / \mathrm{H}^{+}$exchanger isoform 1 is neuroprotective in neonatal hypoxic ischemic brain injury. Antioxid Redox Signal 2011;14:1803-1813.

13 Scholz W, Albus U, Counillon L, Gögelein H, Lang HJ, Linz W, Weichert A, Schölkens BA: Protective effects of HOE642, a selective sodium-hydrogen exchange subtype 1 inhibitor, on cardiac ischaemia and reperfusion. Cardiovasc Res 1995;29:260-268.

14 McIlwain KL, Merriweather MY, Yuva-Paylor LA, Paylor R: The use of behavioral test batteries: effects of training history. Physiol Behav 2001;73:705-717.

15 Kheirbek MA, Britt JP, Beeler JA, Ishikawa Y, McGehee DS, Zhuang X: Adenylyl cyclase type 5 contributes to corticostriatal plasticity and striatum-dependent learning. J Neurosci 2009;29:12115-12124.

16 Rasband WS: ImageJ. US Bethesda, National Institutes of Health, 1997-2009.

17 Paxinos G, Halliday GM, Watson C, Koutcherov $\mathrm{Y}$, Wang $\mathrm{H}$ : Atlas of the Developing Mouse Brain at E17.5, P0 and P6, ed 1. Amsterdam, Elsevier, 2007.

18 Wang S, Wu EX, Tam CN, Lau HF, Cheung PT, Khong PL: Characterization of white matter injury in a hypoxic-ischemic neonatal rat model by diffusion tensor MRI. Stroke 2008;39:2348-2353.

19 McQuillen PS, Ferriero DM: Selective vulnerability in the developing central nervous system. Pediatr Neurol 2004;30:227-235.

20 Vannucci SJ, Hurn PD: Gender differences in pediatric stroke: is elevated testosterone a risk factor for boys? Ann Neurol 2009;66: 713-714.
21 Wang S, Wu EX, Cai K, Lau HF, Cheung PT, Khong PL: Mild hypoxic-ischemic injury in the neonatal rat brain: longitudinal evaluation of white matter using diffusion tensor MR imaging. AJNR Am J Neuroradiol 2009; 30:1907-1913.

22 Chan KC, Khong PL, Lau HF, Cheung PT, Wu EX: Late measures of microstructural alterations in severe neonatal hypoxic-ischemic encephalopathy by MR diffusion tensor imaging. Int J Dev Neurosci 2009;27:607615.

23 Chahboune H, Ment LR, Stewart WB, Rothman DL, Vaccarino FM, Hyder F, Schwartz ML: Hypoxic injury during neonatal development in murine brain: correlation between in vivo DTI findings and behavioral assessment. Cereb Cortex 2009;19:28912901.

24 Kohlhauser C, Mosgöller W, Höger H, Lubec B: Myelination deficits in brain of rats following perinatal asphyxia. Life Sci 2000;67: 2355-2368.

25 Pereira LO, Arteni NS, Petersen RC, da Rocha AP, Achaval M, Netto CA: Effects of daily environmental enrichment on memory deficits and brain injury following neonatal hypoxia-ischemia in the rat. Neurobiol Learn Mem 2007;87:101-108.

26 Miki K, Ishibashi S, Sun L, Xu H, Ohashi W, Kuroiwa T, Mizusawa H: Intensity of chronic cerebral hypoperfusion determines white/ gray matter injury and cognitive/motor dysfunction in mice. J Neurosci Res 2009;87: 1270-1281.

27 Zwicker JG, Missiuna C, Boyd LA: Neural correlates of developmental coordination disorder: a review of hypotheses. J Child Neurol 2009;24:1273-1281.
28 Giedd JN, Castellanos FX, Casey BJ, Kozuch P, King AC, Hamburger SD, Rapoport JL: Quantitative morphology of the corpus callosum in attention deficit hyperactivity disorder. Am J Psychiatry 1994;151:665-669.

29 Wang LW, Chang YC, Lin CY, Hong JS, Huang CC: Low-dose lipopolysaccharide selectively sensitizes hypoxic ischemia-induced white matter injury in the immature brain. Pediatr Res 2010;68:41-47.

30 Carty ML, Wixey JA, Colditz PB, Buller KM: Post-insult minocycline treatment attenuates hypoxia-ischemia-induced neuroinflammation and white matter injury in the neonatal rat: a comparison of two different dose regimens. Int J Dev Neurosci 2008;26: 477-485.

31 Rezaie P, Dean A: Periventricular leukomalacia, inflammation and white matter lesions within the developing nervous system. Neuropathology 2002;22:106-132.

32 Hedtjärn M, Mallard C, Arvidsson P, Hagberg $\mathrm{H}$ : White matter injury in the immature brain: role of interleukin-18. Neurosci Lett 2005;373:16-20.

33 Johnston MV, Ferriero DM, Vannucci SJ, Hagberg H: Models of cerebral palsy: which ones are best? J Child Neurol 2005;20:984987.

34 Liu Y, Kintner DB, Chanana V, Algharabli J, Chen X, Gao Y, Chen J, Ferrazzano P, Olson JK, Sun D: Activation of microglia depends on $\mathrm{Na}^{+} / \mathrm{H}^{+}$exchange-mediated $\mathrm{H}^{+}$homeostasis. J Neurosci 2010;30:15210-15220.

35 Bulle KM, Carty ML, Reinebrant HE, Wixey JA: Minocycline: a neuroprotective agent for hypoxic-ischemic brain injury in the neonate? J Neurosci Res 2009;87:599-608. 